\title{
SOUNDING OPEN CLUSTERS: ASTEROSEISMIC CONSTRAINTS FROM KEPLER ON THE PROPERTIES OF NGC 6791 AND NGC 6819
}

\author{
Sarbani Basu ${ }^{1}$, Frank Grundahl ${ }^{2}$, Dennis Stello ${ }^{3}$, Thomas Kallinger ${ }^{4}, 5$, Saskia HekKer ${ }^{6}$, Benoit Mosser ${ }^{7}$, \\ Rafael A. García ${ }^{8}$, Savita Mathur ${ }^{9}$, Karsten Brogaard $^{2}$, Hans Bruntt $^{2}$, William J. Chaplin ${ }^{6}$, Ning Gai $^{10,1}$, \\ Yvonne Elsworth $^{6}$, Lisa Esch ${ }^{1}$, Jerome Ballot ${ }^{11}$, Timothy R. Bedding ${ }^{3}$, Michael Gruberbauer $^{12}$, Daniel Huber $^{3}$, \\ Andrea Miglio $^{13}$, Mutlu Yildiz ${ }^{14}$, Hans KJeldsen ${ }^{2}$, Jørgen Christensen-DalsgaARd ${ }^{2}$, Ronald L. Gilliland ${ }^{15}$, \\ Michael M. Fanelli ${ }^{16}$, Khadeejah A. Ibrahim ${ }^{17}$, and JefFrey C. Smith ${ }^{18}$ \\ ${ }^{1}$ Department of Astronomy, Yale University, P.O. Box 208101, New Haven, CT 06520-8101, USA; sarbani.basu@ yale.edu \\ ${ }^{2}$ Department of Physics and Astronomy, Aarhus University, 8000 Aarhus C, Denmark \\ ${ }^{3}$ Sydney Institute for Astronomy (SIfA), School of Physics, University of Sydney, NSW 2006, Australia \\ ${ }^{4}$ Department of Physics and Astronomy, University of British Columbia, 6224 Agricultural Road, Vancouver, BC V6T 1Z1, Canada \\ ${ }^{5}$ Institute for Astronomy, University of Vienna, Türkenschanzstrasse 17, 1180 Vienna, Austria \\ ${ }^{6}$ School of Physics and Astronomy, University of Birmingham, Edgbaston, Birmingham B15 2TT, UK \\ ${ }^{7}$ LESIA, CNRS, Université Pierre et Marie Curie, Université Denis Diderot, Observatoire de Paris, 92195 Meudon, France \\ ${ }^{8}$ Laboratoire AIM, CEA/DSM-CNRS, Université Paris 7 Diderot, IRFU/SAp, Centre de Saclay, 91191 Gif-sur-Yvette, France \\ ${ }^{9}$ High Altitude Observatory, National Center for Atmospheric Research, Boulder, CO 80307, USA \\ ${ }^{10}$ Department of Astronomy, Beijing Normal University, Beijing 100875, China \\ ${ }^{11}$ Laboratoire d'Astrophysique de Toulouse-Tarbes, Université de Toulouse, CNRS, 31400 Toulouse, France \\ 12 Institute for Computational Astrophysics, Department of Astronomy and Physics, Saint Marys University, Halifax, NS B3H 3C3, Canada \\ ${ }^{13}$ Institut d'Astrophysique et Géophysique de l'Université de Liège, Allée du six Août, 17 B-4000 Liège, Belgium \\ ${ }^{14}$ Department of Astronomy and Space Sciences, Ege University, Bornova, 35100 İzmir, Turkey \\ ${ }^{15}$ Space Telescope Science Institute, 3700 San Martin Drive, Baltimore, MD 21218, USA \\ ${ }^{16}$ Bay Area Environmental Research Inst./NASA Ames Research Center, Moffett Field, CA 94035, USA \\ ${ }_{17}$ Orbital Sciences Corporation/NASA Ames Research Center, Moffett Field, CA 94035, USA \\ ${ }^{18}$ SETI Institute/NASA Ames Research Center, Moffett Field, CA 94035, USA \\ Received 2011 January 5; accepted 2011 January 25; published 2011 February 9
}

\begin{abstract}
We present initial results on some of the properties of open clusters NGC 6791 and NGC 6819 derived from asteroseismic data obtained by NASA's Kepler mission. In addition to estimating the mass, radius, and log $g$ of stars on the red giant branch (RGB) of these clusters, we estimate the distance to the clusters and their ages. Our model-independent estimate of the distance modulus of NGC 6791 is $(m-M)_{0}=13.11 \pm 0.06$. We find $(m-M)_{0}=11.85 \pm 0.05$ for NGC 6819. The average mass of stars on the RGB of NGC 6791 is $1.20 \pm 0.01 M_{\odot}$, while that of NGC 6819 is $1.68 \pm 0.03 M_{\odot}$. It should be noted that we do not have data that cover the entire RGB and the actual mass will be somewhat lower. We have determined model-dependent estimates of ages of these clusters. We find ages between 6.8 and 8.6 Gyr for NGC 6791, however, most sets of models give ages around 7 Gyr. We obtain ages between 2 and 2.4 Gyr for NGC 6819.
\end{abstract}

Key words: open clusters and associations: individual (NGC 6819, NGC 6791) - stars: fundamental parameters stars: interiors - stars: oscillations

Online-only material: color figures, machine-readable table

\section{INTRODUCTION}

Oscillations in red giant stars in the field have been studied with Kepler (Bedding et al. 2010; Huber et al. 2010; Kallinger et al. 2010b). The first 34 days of science data from NASA's Kepler mission (Borucki et al. 2010) had shown that oscillations of red giant stars in the clusters can also be detected (Stello et al. 2010a). Kepler has been continuously observing stars in NGC 6819 and NGC 6791 for more than a year. In this Letter, we present results of asteroseismic analyses of red giant stars in NGC 6791 and NGC 6819 to determine some basic properties of these clusters. In particular, we derive model-independent estimates of the distance moduli of these clusters as well as the average mass of the stars on the red giant branch (RGB). We also derive estimates of ages of these stars. We use Kepler observations made between 2009 May and 2009 December (i.e., Q1-Q3 data) for this work.

The stars in these clusters, particularly those in NGC 6791, are faint, and currently available Kepler data allow us to extract basic asteroseismic parameters of only the bright RGB and helium- core-burning red-clump stars. Although red giant stars have relatively large uncertainties in asteroseismically determined properties, the uncertainties are still small enough to make such an analysis viable. The fact that all cluster stars can be assumed to have the same distance and age increases the precision of our results.

\section{DATA AND ANALYSIS}

We studied 34 stars in NGC 6791 and 21 stars in NGC 6819. Only confirmed members were selected (see Stello et al. 2010a, 2010b). The stars studied in this work are shown in Figure 1. Note that although oscillation characteristics have been detected and measured in red clump stars of both clusters, we do not use them in this study for reasons explained later. Since red giant oscillations are slow, Kepler long-cadence observations ( $\Delta t \simeq 30$ minutes; Jenkins et al. 2010) are adequate for our work. The time series for each star was analyzed by five different pipelines: SYD (Huber et al. 2009), COR (Mosser \& Appourchaux 2009), CAN (Kallinger et al. 2010a), A2Z (Mathur et al. 2010), and OCT (Hekker et al. 2010). A 


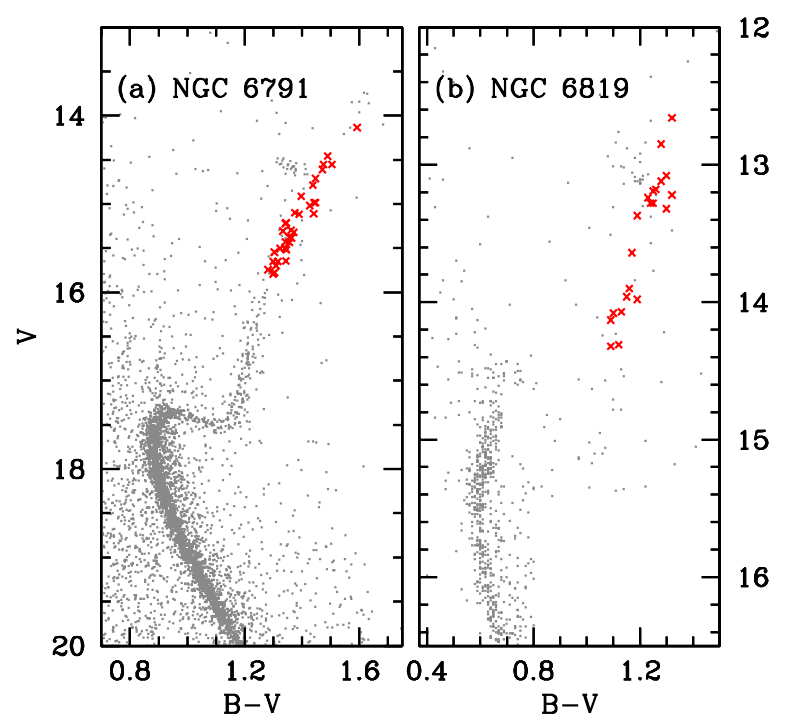

Figure 1. Color-magnitude diagrams of NGC 6791 and NGC 6819 with the stars used in this work plotted in red. Photometry data for NGC 6791 are from Stetson et al. (2003) and for NGC 6819 from Hole et al. (2009).

(A color version of this figure is available in the online journal.)

comparison of these pipelines can be found in Hekker et al. (2011a). We use two asteroseismic parameters in this study: the so-called large frequency separation, $\Delta v$, and the frequency of maximum power $v_{\max }$. Each pipeline produced estimates of these parameters. For each cluster we adopted the results from the pipeline that consistently gave the closest result to the average of the results obtained by all pipelines. The uncertainties in $\Delta v$ and $v_{\max }$ returned by the pipeline were increased to reflect the variation of $\Delta v$ and $v_{\max }$ values returned by the different pipelines. We used those stars for which all pipelines returned a result. Details of the data preparation and analysis can be found in Hekker et al. (2011b).

The large separation $\Delta v$ depends on the mean density of a star (Christensen-Dalsgaard 1988):

$$
\frac{\Delta v}{\Delta v_{\odot}}=\sqrt{\frac{M / M_{\odot}}{\left(R / R_{\odot}\right)^{3}}} .
$$

The scaling holds to within $3 \%$ over most of the H-R diagram (Stello et al. 2009a). The frequency of maximum power in the oscillations power spectrum, $v_{\max }$, is related to the acoustic cutoff frequency of a star (e.g., see Kjeldsen \& Bedding 1995; Bedding \& Kjeldsen 2003; Chaplin et al. 2008) and scales as

$$
\frac{v_{\max }}{v_{\max , \odot}}=\frac{M / M_{\odot}}{\left(R / R_{\odot}\right)^{2} \sqrt{\left(T_{\text {eff }} / T_{\text {eff }, \odot}\right)}} .
$$

Data from CoRoT have verified this scaling (Mosser et al. 2010). Thus if $\Delta v, v_{\max }$, and $T_{\text {eff }}$ are known, Equations (1) and (2) represent two equations in two unknowns $(M$ and $R$ ) and can be solved to obtain $M, R$, and $g$. However, Equations (1) and (2) are not constrained by the equations of stellar structure and assume that all values of $T_{\text {eff }}$ are possible for a star of a given mass and radius resulting in unrealistically large uncertainties in the derived mass and radius. We overcame this by using socalled grid-based methods, where the characteristics of stars are determined by searching among the models to get a "best fit" for a given observed set of $\Delta v, v_{\max }, T_{\text {eff }}$, and $[\mathrm{Fe} / \mathrm{H}]$. Gai et al. (2011) have shown that grid-based seismology produces model-independent results for $R$ and $\log g$; there are small biases in $M$ that can be minimized if metallicity is known, and that the increased precision is worth the small model dependence in mass.

In this work we used three different implementations of gridbased methods - the Yale-Birmingham (YB) pipeline (Basu et al. 2010; Gai et al. 2011), a slightly modified version of the RADIUS pipeline of Stello et al. (2009b), and the Seismic Fundamental Parameter (SFP) pipeline of Kallinger et al. (2010b). YB was used with four different sets of models. One set, described by Gai et al. (2011), was based on YREC (Demarque et al. 2008). The other sets were those of Dotter et al. (2008), Marigo et al. (2008), and models from the Yonsei-Yale (YY) isochrones (Demarque et al. 2004). Additionally, a set of YREC models with $Y=0.30$ was also used to analyze NGC 6791 data because of suggestions that the helium abundance of NGC 6791 is high (Demarque et al. 1992; Brogaard et al. 2011). The RADIUS pipeline uses models described by Stello et al. (2009b) that were constructed with the ASTEC code (Christensen-Dalsgaard 2008). The SFP pipeline is based on the BASTI models (Pietrinferni et al. 2004). The different model grids use different physics inputs. While the YREC and Dotter et al. models include gravitational settling and diffusion of helium and heavy elements, YY models only include diffusion of helium. The BASTI models, the Marigo et al. models, and the Stello et al. models do not incorporate settling and diffusion of elements at all. Nuclear reaction rates and equations of state used to construct the models are different too. The mixing length parameter for models in each grid was calibrated to a standard solar model constructed using the corresponding stellar evolution code and it differs somewhat from grid to grid because of differences in the physics used. Equations (1) and (2) were used to calculate $\Delta v$ and $v_{\max }$ for the models in all grids. Since the ASTEC and YY grids do not include core-helium-burning red-clump stars, we restricted our study to stars that are not likely to be in the core-helium-burning phase.

Effective temperatures for stars in the two clusters were derived using the color-temperature calibrations of Ramírez \& Meléndez (2005). All targets are sufficiently bright to have $J H K$ photometry from Two Micron All Sky Survey (Skrutskie et al. 2006), allowing us to determine the temperatures based on the $(V-K)$ colors. We used $V$ estimates of Stetson et al. (2003) for NGC 6791 targets and Hole et al. (2009) for NGC 6819 targets. Brogaard et al. (2011) estimated the reddening toward NGC 6791 to be $E(B-V)=0.16 \pm 0.02$ and that is the value adopted by us. We adopted a value of $E(B-V)=0.15$ (Bragaglia et al. 2001) for NGC 6819. The uncertainty in each temperature estimate is believed to be about $100 \mathrm{~K}$ and arises from uncertainties in photometry, reddening, as well as uncertainty in the color-temperature relationship.

We adopted a metallicity of $[\mathrm{Fe} / \mathrm{H}]=+0.29$ for NGC 6791 (Brogaard et al. 2011) and +0.09 for NGC 6819 (Bragaglia et al. 2001). We have assumed uncertainties of $0.1 \mathrm{dex}$, which is somewhat larger than the internal errors claimed by each group.

\section{RESULTS AND DISCUSSION}

For each cluster we first present estimates of stellar properties that are nearly model independent, before looking at the question of age estimates which are known to be model dependent. As in the case of the basic seismic inputs $\Delta v$ and $v_{\max }$, we only use the results of one of the pipeline-grid combination for all parameters except age. The selected pipeline-grid combination 

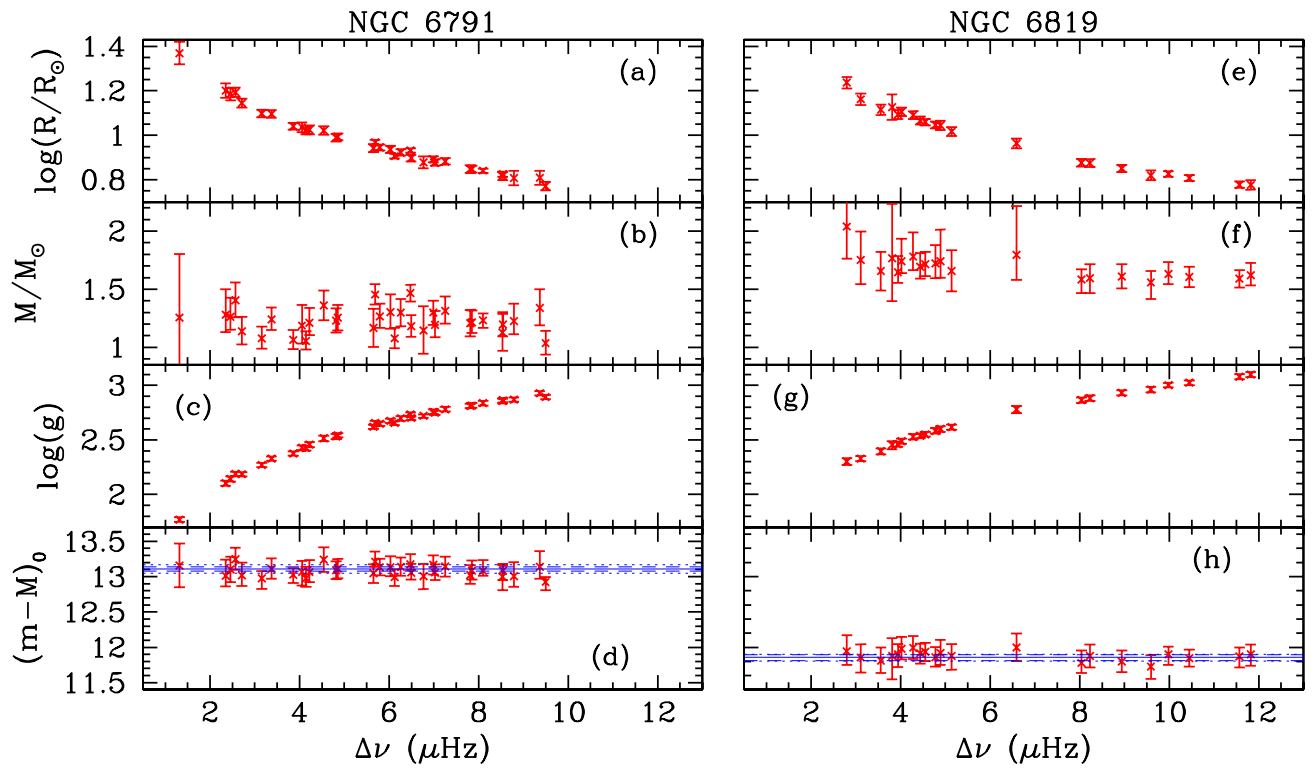

Figure 2. Derived properties of stars in NGC 6791 (left panels) and NGC 6819 (right panels) plotted against their large separations. We show the results for radius (panels (a) and (e)), mass (panels (b) and (f)), $\log g$ (panels (c) and (g)), and extinction-corrected distance modulus (panels (d) and (h)) for the individual stars. The distance modulus to the cluster has also been determined by adopting the prior that all stars have to have the same distance modulus. The results are shown as the solid horizontal line. The dashed line shows the $1 \sigma$ limits of the random error, and the dotted lines show the result when the systematic error caused by metallicity and reddening are added to the random error. A simple average of the DMs of individual stars gives a similar result, however, the uncertainties in the mean are somewhat larger than the $1 \sigma$ limit shown by the dashed lines.

(A color version of this figure is available in the online journal.)

Table 1

Properties of Stars in NGC 6791 and NGC 6819

\begin{tabular}{lccccccc}
\hline \hline KIC ID & $\begin{array}{c}T_{\text {eff }} \\
(\mathrm{K})\end{array}$ & $\begin{array}{c}V \\
(\mathrm{mag})\end{array}$ & $\begin{array}{c}\Delta v \\
(\mu \mathrm{Hz})\end{array}$ & $\begin{array}{c}v_{\max } \\
(\mu \mathrm{Hz})\end{array}$ & $\begin{array}{c}\text { Radius } \\
\left(R_{\odot}\right)\end{array}$ & $\begin{array}{c}\text { Mass } \\
\left(M_{\odot}\right)\end{array}$ & $\begin{array}{c}\log g \\
(\mathrm{cgs})\end{array}$ \\
\hline \multicolumn{7}{c}{ NGC 6791} \\
\hline 2437340 & 4007 & 14.135 & $1.317 \pm 0.054$ & $8.028 \pm 0.257$ & $23.40_{-2.86}^{+2.90}$ & $1.26_{-0.53}^{+0.55}$ & $1.767_{-0.017}^{+0.026}$ \\
2437444 & 4186 & 14.553 & $2.459 \pm 0.073$ & $18.658 \pm 0.496$ & $15.32_{-0.01}^{+1.017}$ & $1.26_{-0.11}^{+0.16}$ & $2.142_{-0.023}^{+0.022}$ \\
2437816 & 4215 & 14.459 & $2.348 \pm 0.100$ & $16.948 \pm 0.484$ & $15.88_{-0.61}^{+1.23}$ & $1.28_{-0.15}^{+0.22}$ & $2.104_{-0.025}^{+0.021}$ \\
2437507 & 4246 & 14.554 & $2.568 \pm 0.052$ & $20.654 \pm 0.282$ & $15.60_{-0.77}^{+0.75}$ & $1.40_{-0.14}^{+0.16}$ & $2.189_{-0.020}^{+0.020}$ \\
\hline & & & \multicolumn{7}{c}{ NGC 6819} \\
\hline 5112880 & 4443 & 12.66 & $2.800 \pm 0.050$ & $26.650 \pm 1.065$ & $17.25_{-1.04}^{+1.05}$ & $2.04_{-0.28}^{+0.35}$ & $2.302_{-0.031}^{+0.031}$ \\
4937576 & 4481 & 13.08 & $3.560 \pm 0.051$ & $32.290 \pm 1.516$ & $13.03_{-0.76}^{+0.73}$ & $1.66_{-0.17}^{+0.16}$ & $2.395_{-0.026}^{+0.026}$ \\
5023732 & 4512 & 12.85 & $3.110 \pm 0.032$ & $27.450 \pm 1.234$ & $14.52_{-0.85}^{+0.88}$ & $1.75_{-0.21}^{+0.24}$ & $2.329_{-0.023}^{+0.024}$ \\
5113041 & 4521 & 13.18 & $3.940 \pm 0.051$ & $37.570 \pm 1.384$ & $12.39_{-0.51}^{+0.59}$ & $1.65_{-0.09}^{+0.14}$ & $2.461_{-0.022}^{+0.022}$ \\
5112734 & 4528 & 13.19 & $4.020 \pm 0.050$ & $40.210 \pm 1.545$ & $12.76_{-0.62}^{+0.57}$ & $1.74_{-0.10}^{+0.20}$ & $2.489_{-0.024}^{+0.023}$ \\
\hline
\end{tabular}

Note. Stars are sorted according to $T_{\text {eff }}$. The assumed uncertainty in temperature is $100 \mathrm{~K}$.

(This table is available in its entirety in a machine-readable form in the online journal. A portion is shown here for guidance regarding its form and content.)

was the one that produced results that were consistently the closest to the average of the results obtained from all pipelines and grids of models. For each parameter of each star, the spread in results obtained from the different pipelines and grids were added in quadrature to the formal uncertainty obtained from the selected pipeline-grid combination. The basic data for both clusters are listed in Table 1.

\subsection{NGC 6791}

Results on temperature, radius, mass, $\log g$, and distance modulus (DM) for the stars in NGC 6791 are shown in Figures 2(a)-(d). The results are also listed in Table 1. The results are derived with the adopted value of metallicity $([\mathrm{Fe} / \mathrm{H}]=+0.29)$ and reddening $(E(B-V)=0.16)$.
The stars in the sample cover a temperature range of approximately $4000-4600 \mathrm{~K}$. The radii of the stars increase almost monotonically from about $6 R_{\odot}$ at the highest temperatures to about $24 R_{\odot}$ at the lowest temperatures. This trend is completely consistent with what we expect from standard red giant models. Although asteroseismic radius estimates of giants have large uncertainties (compared to other stars), we can still get radii to a precision of about $5 \%$ for all but six stars. Of the rest, five have precisions better to $8 \%$ and one star has a $\sim 12 \%$ uncertainty. The large uncertainty in this case is a reflection of the spread in the results obtained by different pipelines; we need more data to resolve this.

Within uncertainties, all the stars have roughly equal masses. The formal averaged mass for the stretch of the RGB branch 
for which we have data is $1.20 \pm 0.01 M_{\odot}$. The uncertainty on the individual masses is larger, though generally less than $10 \%$. Better estimates of masses will be possible once we model the detailed oscillation spectrum of each star. The data available so far do not yet allow us to do that.

We obtain precise results for $\log g$-with uncertainties less than $1 \%$ for most stars. Given that spectroscopic estimates of $\log g$ for giants is uncertain, asteroseismology gives us an alternative method of determining this quantity. Since $\log g$ estimates are required to determine stellar temperatures from spectra, this should enable us to obtain better temperature estimates of these stars.

NGC 6791 has been an object of repeated investigations ever since the first in-depth study by Kinman (1965). There have been many estimates of the distance to this cluster. Kinman (1965) found that $(m-M)_{0}=13.55 \mathrm{mag}$. Since then different studies have resulted in a wide range of values for the DM that span the range of 12.6 mag (Anthony-Twarog \& Twarog 1985 ) to 13.6 mag (Harris \& Canterna 1981). Discussions of this can be found in Stetson et al. (2003) and Carraro et al. (2006). Our model-independent estimates of radius, along with temperature estimates, allow us to determine the DM of the stars in the cluster. Better results are obtained if, instead of calculating luminosity explicitly from radius and $T_{\text {eff }}$, we again do a grid search to determine luminosity, or the absolute visual magnitude directly. Most of the grids include $M_{V}$ calculated from $L$ using a variety of color conversions. Where $M_{V}$ was not available in the grid, the luminosity was converted to $M_{V}$ using the color table of Lejeune et al. (1997).

The DMs of our sample of NGC 6791 stars are shown in Figure 2(e). Also shown is the DM obtained assuming that all stars are at the same distance. Using an extinction of $A_{V}=3.1 E(B-V)$, we obtain $(m-M)_{0}=13.11 \pm 0.06$ for NGC 6791 assuming $[\mathrm{Fe} / \mathrm{H}]=+0.29$. There is a small dependence of the DM on the adopted value of $E(B-V)$ and metallicity mainly through differences in temperature estimates and that can be seen in Table 2. All estimates of the DM are very similar and the adopted value of $E(B-V)$ does not change the DM drastically. Much of the older literature on NGC 6791 finds/adopts $E(B-V) \simeq 0.1$ which is smaller than our adopted value of $E(B-V)=0.16$.

\subsection{NGC 6819}

Results for NGC 6819 are shown in Figures 2(e)-(h) and also listed in Table 1 . The stars in our sample cover a temperature range of $4450-4850 \mathrm{~K}$. The radii of the stars range from 6 to $17 R_{\odot}$ and with precisions to better than $5 \%$ for all but three stars and better than $6 \%$ for all except one star. The average mass of these stars is $1.68 \pm 0.03 M_{\odot}$. The uncertainty on individual mass estimates is less than $10 \%$ in most cases. The higher mass of these stars compared to those of NGC 6791 is indicative of a lower age for this cluster. Again the uncertainty in $\log g$ is less than $1 \%$ in most cases.

The extinction-corrected DM for this cluster, when we assume $[\mathrm{Fe} / \mathrm{H}]=+0.09$ and $E(B-V)=0.15$, is $(m-M)_{0}=$ $11.85 \pm 0.05$. The DM, for the same value of metallicity is 11.87 for $E(B-V)=0.10$ and 11.83 for $E(B-V)=0.20$. The $E(B-V)$ ranges were selected from values used in literature about this cluster. If we assume the same reddening but $[\mathrm{Fe} / \mathrm{H}]=-0.10$ then we obtain $(m-M)_{0}=11.91$. Thus, as with NGC 6791, we can obtain precise values of the DM even in the presence of some of the uncertainties in reddening. Changing metallicity by larger than the assumed uncertainty
Table 2

Dependence of DM and Age on $E(B-V)$ and $[\mathrm{Fe} / \mathrm{H}]$

\begin{tabular}{lccc}
\hline \hline & \multicolumn{3}{c}{$\mathrm{NGC} 6791$} \\
$E(B-V)$ & {$[\mathrm{Fe} / \mathrm{H}]$} & $(m-M)_{0}$ & Age $(\mathrm{Gyr})$ \\
\hline 0.10 & +0.29 & $13.22 \pm 0.06$ & $9.2_{-0.5}^{+0.4}$ \\
0.125 & +0.29 & $13.17 \pm 0.06$ & $8.7_{-0.5}^{+0.4}$ \\
0.16 & +0.29 & $13.11 \pm 0.06$ & $8.0_{-0.5}^{+0.5}$ \\
0.18 & +0.29 & $13.09 \pm 0.06$ & $7.6_{-0.5}^{+0.5}$ \\
0.20 & +0.29 & $13.04 \pm 0.06$ & $7.2_{-0.5}^{+0.5}$ \\
0.25 & +0.29 & $12.98 \pm 0.07$ & $6.0_{-0.6}^{+0.5}$ \\
0.16 & +0.25 & $13.13 \pm 0.06$ & $8.1_{-0.5}^{+0.5}$ \\
0.16 & +0.29 & $13.11 \pm 0.06$ & $8.0_{-0.5}^{+0.5}$ \\
0.16 & +0.35 & $13.07 \pm 0.06$ & $7.9_{-0.5}^{+0.5}$ \\
0.16 & +0.40 & $13.04 \pm 0.06$ & $7.8_{-0.5}^{+0.5}$ \\
\hline & & $\mathrm{NGC} 6819$ & \\
$E(B-V)$ & {$[\mathrm{Fe} / \mathrm{H}]$} & $(m-M)_{0}$ & Age $(\mathrm{Gyr})$ \\
\hline 0.10 & +0.10 & $11.87 \pm 0.05$ & $2.99_{-0.25}^{+0.50}$ \\
0.125 & +0.10 & $11.86 \pm 0.05$ & $2.58_{-0.21}^{+0.41}$ \\
0.15 & +0.10 & $11.85 \pm 0.05$ & $2.23_{-0.17}^{+0.31}$ \\
0.175 & +0.10 & $11.85 \pm 0.05$ & $1.94_{-0.14}^{+0.24}$ \\
0.20 & +0.10 & $11.83 \pm 0.06$ & $1.70_{-0.12}^{+0.18}$ \\
0.15 & -0.15 & $11.92 \pm 0.05$ & $2.39_{-0.17}^{+0.41}$ \\
0.15 & -0.10 & $11.91 \pm 0.04$ & $2.36_{-0.17}^{+0.39}$ \\
0.15 & -0.05 & $11.88 \pm 0.05$ & $2.33_{-0.17}^{+0.36}$ \\
0.15 & 0.00 & $11.89 \pm 0.04$ & $2.30_{-0.17}^{+0.34}$ \\
0.15 & +0.05 & $11.86 \pm 0.05$ & $2.27_{-0.17}^{+0.33}$ \\
0.15 & +0.10 & $11.85 \pm 0.05$ & $2.23_{-0.17}^{+0.31}$ \\
0.15 & +0.15 & $11.84 \pm 0.05$ & $2.18_{-0.17}^{+0.30}$ \\
\hline & & &
\end{tabular}

does change the obtained value, but not by much. The $E(B-V)$ and $[\mathrm{Fe} / \mathrm{H}]$ dependence can be seen in Table 2 .

The DM values we have derived are somewhat smaller than those discussed in literature in the context of isochrone fitting. Kalirai \& Tosi (2004) used $(m-M)_{0}$ of 12-12.2 mag. Hole et al. (2009) used $(m-M)_{V}=12.3 \mathrm{mag}$, which for their adopted reddening of $E(B-V)=0.1 \mathrm{mag}$ is still somewhat larger than what we find.

$$
\text { 3.3. Ages }
$$

The two easily observed asteroseismic quantities, $\Delta v$ and $v_{\max }$, do not have a direct dependence on age. Age estimates using these two quantities thus rely on models. For individual stars, the grid method gives large uncertainties, around $25 \%$ for most, but even larger for some. However, it can be shown that applying an equal-age prior to all stars in a given cluster allows ages to be determined quite precisely (Gai et al. 2011). Unlike the case of isochrone fitting, we can derive the age of the cluster without turn-off stars and the estimates are independent of distance. Dependence on reddening comes through temperature calibrations. Of the three pipelines used, at the moment only YB allows the use of the prior of identical ages and hence only those results are discussed. Figure 3 shows the ages of the individual stars in the two clusters as obtained with YY models. Also shown is the age determined when the prior of identical age is applied. As is clear, the uncertainties for individual stars is much larger than uncertainties for the cluster as a whole.

As expected from previous studies, our results show that NGC 6791 is considerably older than NGC 6819. For the assumed metallicity of $[\mathrm{Fe} / \mathrm{H}]=+0.29$, we obtain fairly 

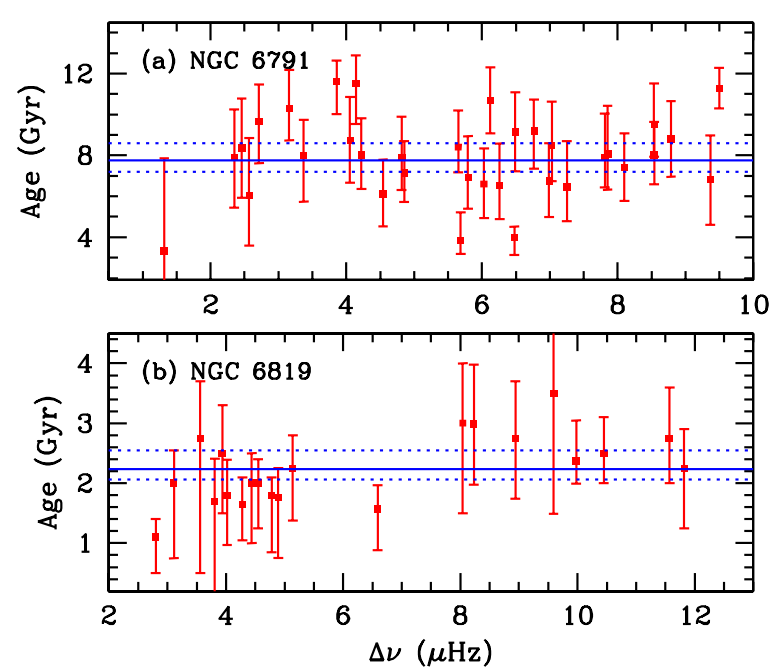

Figure 3. Derived ages of stars in NGC 6791 (panel (a)) and NGC 6819 (panel (b)) using YY models. The points with error bars show the results for individual stars while the horizontal lines in each panel is the result obtained applying the prior that all stars in the cluster have the same age.

(A color version of this figure is available in the online journal.)

consistent results from all the model grids used. The highest age obtained is $8.6 \pm 0.5 \mathrm{Gyr}$ for YREC models, though the YREC models with the high helium abundance gives $7.3_{-0.4}^{+0.7}$ Gyr. We obtain $8.0_{-0.6}^{+0.8}$ Gyr using YY, and 7.2 ${ }_{-0.4}^{+0.8}$ using Dotter et al. models. Marigo et al. (2008) models give a lower age: $6.8 \pm$ 0.4 Gyr. The difference between the models lie in the helium abundance used as well as in the mixing length parameter used and the details of core overshoot. For any given grid of models, changing the metallicity changes the estimated age, but by far the larger change occurs when the assumed reddening is changed since that changes the estimated temperatures. Table 2 lists the age estimates using YY models for different values of $E(B-V)$ and $[\mathrm{Fe} / \mathrm{H}]$. Thus, it is crucial to estimate reddening precisely in order to derive a definitive age estimate for this cluster.

Our age estimates for NGC 6791 lie somewhat on the lower side of most isochrone-fitting-based estimates. Previous studies give a wide range of ages between 8 and 12 Gyr (e.g., Carraro et al. 1994, 2006; Chaboyer et al. 1999; Carney et al. 2005). Among the more recent studies are those of Carraro et al. (2006), who concluded that the age of this cluster should be in the range 7.5-8.5 Gyr with preference given to the higher limit. Our estimated ages are, however, consistent with the age estimate of 7 Gyr obtained by Brogaard et al. (2011). This consistency is probably the result of our adopting their value of $E(B-V)$. Spectroscopic temperature estimates of stars in this cluster are required to resolve this issue. Although our derived age is lower than most, it is not low enough to alleviate the problem that this old metal-rich cluster poses for scenarios of Galactic chemical evolution.

Unlike the case of NGC 6791, our estimates for the age of NGC 6819 agree with those determined through isochrone fitting. We derive ages between 2.1 and $2.5 \mathrm{Gyr}$. Use of YREC models results in an age estimate of $2.38_{-0.20}^{+0.28} \mathrm{Gyr}$. YY gives $2.23_{-0.17}^{+0.31}$ Gyr. We found 2.31 ${ }_{-0.16}^{+0.30}$ Gyr with Dotter et al. (2008) models and $2.00_{-0.14}^{+0.21} \mathrm{Gyr}$ with Marigo et al. (2008) models. As with NGC 6791, the age estimates depend on the adopted value of $E(B-V)$. The estimated age increases if we decrease the assumed $E(B-V)$. The estimated age is also sensitive to metallicity and the dependence of age derived from YY models on $E(B-V)$ and $[\mathrm{Fe} / \mathrm{H}]$ is listed in Table 2. Ages in literature vary from 1.8 to $2.5 \mathrm{Gyr}$ (Kalirai \& Tosi 2004; Hole et al. 2009). We should be able to place much tighter constraints on the age of this cluster when we have data on individual frequencies of subgiant stars in this cluster. The oscillation spectrum of subgiant stars is extremely sensitive to age, and modeling the spectra gives more precise, though still somewhat model-dependent, age estimates (see, e.g., Metcalfe et al. 2010).

\section{CONCLUSIONS}

Early seismic data from Kepler has allowed us to determine the basic properties of some of the red giants in NGC 6791 and NGC 6819, which in turn has enabled us to determine the distance to those clusters in a model-independent manner. The final uncertainties are likely to improve as we get data on more stars, thus these results are just a foretaste of how we can use Kepler data to do high-precision studies of clusters.

Age estimates of the clusters are still somewhat uncertain, and the adopted value of reddening plays a crucial role through its effect on temperature estimates. We have, however, demonstrated that with only a handful of stars it is possible to derive ages of clusters to levels of precision that rival ages obtained through isochrone fitting of the entire color-magnitude diagram of a cluster. This holds promise for future studies of the two sparse clusters, NGC 6811 and NGC 6866, in the Kepler field. For more reliable age determinations, we need better estimates of the effective temperature of cluster stars. It would also help to have independent estimates of the mass of some stars in binary systems, since that would constrain the search space.

The stars in these clusters are still being observed and we should soon have time series that are long enough for us to be able to extract individual frequencies. Modeling the frequency spectrum of each under the constraint that all stars need to be modeled with the same metallicity and that they should have the same age will provide more stringent constraints on the properties of these clusters.

Funding for the Kepler mission is provided by NASA's Science Mission Directorate. The authors wish to thank the entire Kepler team, without whom these results would not be possible. We also thank all funding councils and agencies that have supported the activities of Working Group 2 of the Kepler Asteroseismic Science Consortium.

Facility: Kepler

\section{REFERENCES}

Anthony-Twarog, B. J., \& Twarog, B. A. 1985, ApJ, 291, 595

Basu, S., Chaplin, W. J., \& Elsworth, Y. 2010, ApJ, 710, 1596

Bedding, T. R., \& Kjeldsen, H. 2003, PASA, 20, 203

Bedding, T. R., et al. 2010, ApJ, 713, L176

Borucki, W. J., Koch, D. G., \& Basri, G. 2010, Science, 327, 977

Bragaglia, A., et al. 2001, AJ, 121, 327

Brogaard, K., Bruntt, H., Grundahl, F., Clausen, J. V., Frandsen, S., VandenBerg, D. A., \& Bedin, L. R. 2011, A\&A, 525, A2

Carney, B. W., Lee, J. -W., \& Dodson, B. 2005, AJ, 129, 656

Carraro, G., Chiosi, C., Bertelli, G., \& Bressan, A. 1994, A\&AS, 103, 375

Carraro, G., Villanova, S., Demarque, P., McSwain, M. V., Piotto, G., \& Bedin, L. R. 2006, ApJ, 643, 1151

Chaboyer, B., Green, E. M., \& Liebert, J. 1999, AJ, 117, 1360

Chaplin, W. J., Houdek, G., Appourchaux, T., Elsworth, Y., New, R., \& Toutain, T. 2008, A\&A, 485, 813

Christensen-Dalsgaard, J. 1988, in Proc. IAU Symp. 123, Advances in helio- and asteroseismology, ed. J. Christensen-Dalsgaard \& S. Frandsen (Dordrecht: Reidel), 295

Christensen-Dalsgaard, J. 2008, ApS\&S, 316, 13 
Demarque, P., Green, E. M., \& Guenther, D. B. 1992, AJ, 103, 151

Demarque, P., Guenther, D. B., Li, L. H., Mazumdar, A., \& Straka, C. W. 2008, Ap\&SS, 316, 31

Demarque, P., Woo, J. -H., Kim, Y. -C., \& Yi, S. K. 2004, ApJS, 155, 667

Dotter, A., Chaboyer, B., Jeremovic, D., Kostov, V., Baron, E., \& Ferguson, J. W. 2008, ApJS, 178, 89

Gai, N., Basu, S., Chaplin, W. J., \& Elsworth, Y. 2011, ApJ, in press (arXiv: 1009.3018)

Harris, W. E., \& Canterna, R. 1981, AJ, 86, 1332

Hekker, S., et al. 2010, MNRAS, 402, 2049

Hekker, S., et al. 2011a, A\&A, 525, A131

Hekker, S., et al. 2011b, A\&A, submitted

Hole, K. T., Geller, A. M., Mathieu, R. D., Platais, I., Meibom, S., \& Latham, D. W. 2009, AJ, 138, 159

Huber, D., Stello, D., Bedding, T. R., Chaplin, W. J., Arentoft, T., Quirion, P.-O., \& Kjeldsen, H. 2009, Commun. Asteroseismol., 160, 74

Huber, D., et al. 2010, ApJ, 723, 1607

Jenkins, J. M., et al. 2010, ApJ, 713, L120

Kalirai, J. S., \& Tosi, M. 2004, MNRAS, 351, 649

Kallinger, T., et al. 2010a, A\&A, 509, A77
Kallinger, T., et al. 2010b, A\&A, 522,

Kinman, T. D. 1965, ApJ, 142, 655

Kjeldsen, H., \& Bedding, T. R. 1995, A\&A, 293, 87

Lejeune, Th., Cuisinier, F., \& Buser, R. 1997, A\&A, 125, 229

Marigo, P., Girardi, L., Bressan, A., Groenewegen, M. A. T., Silva, L., \& Granato, G. L. 2008, A\&A, 482, 883

Mathur, S., et al. 2010, A\&A, 511, A46

Metcalfe, T. S., et al. 2010, ApJ, 723, 1583

Mosser, B., \& Appourchaux, T. 2009, A\&A, 508, 877

Mosser, B., et al. 2010, A\&A, 517, 22

Pietrinferni, A., Cassisi, S., Salaris, M., \& Castelli, F. 2004, ApJ, 612, 168

Ramírez, I., \& Meléndez, J. 2005, ApJ, 626, 446

Skrutskie, M. F., et al. 2006, AJ, 131, 1163

Stello, D., Chaplin, W. J., Basu, S., Elsworth, Y., \& Bedding, T. R. 2009a, MNRAS, 400, L80

Stello, D., et al. 2009b, ApJ, 700, 1589

Stello, D., et al. 2010a, ApJ, 713, L182

Stello, D., et al. 2010b, submitted

Stetson, P. B., Bruntt, H., \& Grundahl, F. 2003, PASP, 115, 413 